\title{
Necessary voices
}

Given that it is based on heredity and has the ability to trace connections, genetics as a scientific discipline should be among those most attuned to diversity and global perspectives. Indigenous communities and scientists have much to contribute to genetics research, and they are making their voices heard. We celebrate these essential members of our larger genetics community, and we look forward to working together to learn from past examples, meet present challenges and support future opportunities.

T

he first-ever international conference on Indigenous genomics convened in late January in Aotearoa, New Zealand. Organized by the Summer internship for INdigenous peoples in Genomics (SING) Consortium, this meeting brought together representatives and alumni from SING USA, SING Canada, SING Australia and SING Aotearoa, thus supporting appreciation of issues in genomics research that are important not only to particular peoples but also to those spanning Indigenous communities worldwide. Over two days at the beautiful University of Waikato campus, scientists with Indigenous backgrounds presented their work on human health, biobanks, ancient DNA and taonga species (taonga is defined as 'something treasured or valued by Māori' and is often used in relation to indigenous species but can also apply to non-indigenous species), among other topics. There was also ample opportunity for discussion about Indigenous communities' concerns regarding genomics research and the best strategies for navigating the application of rapidly developing technologies in relevant and appropriate ways.

Genetics research is currently in a very exciting time. Next-generation sequencing and CRISPR genome engineering have revolutionized the field. These new technologies have brought with them a host of ethical and, in some cases, even philosophical issues. More importantly, for Indigenous communities, there are specific concerns relating to precision medicine, genome editing, gene drives, ancient-DNA analysis, biocultural labeling, biorepositories, disease genomics and transgenerational epigenetics that might be underappreciated or unknown by non-Indigenous scientists. We of course recognize that Indigenous groups are not a monolith, and that there is a richness and depth to the diversity of different worldviews held by Indigenous peoples across the continents. Even so, there exist commonalities that resonate, particularly regarding genetics. Cultures that place a premium on passed-down knowledge, a reverence for who and what came before, and larger contexts of space and place can have much to teach the larger genetics community.

When discussing these issues, the legacy of colonialism and how it contributes to the health and economic disparities faced by Indigenous communities is impossible to ignore. Because of the potential benefits of genetics research, we as a worldwide community must ensure that no populations are excluded and must make efforts to responsibly work alongside Indigenous researchers and participants, listening to their concerns and ensuring that their interests are prioritized on these projects.

As a journal that aims to serve all members of the genetics community, Nature Genetics pledges to be inclusive in its practices and to support research across all populations and areas. We also recognize the unique talents, ideas and perspectives brought by Indigenous scientists, and that overlooking these would be our loss. Among the many things that we can do to promote diversity, remaining open to input is one of the most important. In addition, we can take concrete steps to ensure that all voices are represented in our activities, by involving Indigenous researchers in every part of the publication process, as authors, reviewers and readers. We can also collaborate on meetings and initiatives surrounding ethical issues in research involving Indigenous participants, such as consent and data ownership. In general, we believe appreciating expertise and reciprocating benefits are important, as is knowing when to listen, when to lead and more importantly when to follow.

These Indigenous voices and perspectives have always been present. The onus now is on the greater genetics community to learn from Indigenous colleagues and proceed in the spirit of partnership and cooperation. We have not fully described here the ideas, perceptions and concerns shared by members of the Indigenous community; we prefer to let them speak for themselves, and we hope to see many of their contributions in these pages in the future.

Published online: 5 February 2020 https://doi.org/10.1038/s41588-020-0585-6 